\title{
Electromagnetic Field Generation in the Downstream of Electrostatic Shocks Due to Electron Trapping
}

\author{
A. Stockem, ${ }^{1,2, *}$ T. Grismayer, ${ }^{2}$ R. A. Fonseca, ${ }^{2,3}$ and L. O. Silva ${ }^{2}$ \\ ${ }^{1}$ Institut für Theoretische Physik, Lehrstuhl IV: Weltraum- und Astrophysik, Ruhr-Universität Bochum, D-44780 Bochum, Germany \\ ${ }^{2}$ GoLP/Instituto de Plasmas e Fusão Nuclear-Laboratório Associado, Instituto Superior Técnico, \\ Universidade de Lisboa, 1049-001 Lisboa, Portugal \\ ${ }^{3}$ ISCTE Instituto Universitário Lisboa, Avenida das Forças Armadas, 1649-026 Lisbon, Portugal
}

(Received 28 January 2014; published 3 September 2014)

\begin{abstract}
A new magnetic field generation mechanism in electrostatic shocks is found, which can produce fields with magnetic energy density as high as 0.01 of the kinetic energy density of the flows on time scales $\sim 10^{4} \omega_{p e}^{-1}$. Electron trapping during the shock formation process creates a strong temperature anisotropy in the distribution function, giving rise to the pure Weibel instability. The generated magnetic field is well confined to the downstream region of the electrostatic shock. The shock formation process is not modified, and the features of the shock front responsible for ion acceleration, which are currently probed in laserplasma laboratory experiments, are maintained. However, such a strong magnetic field determines the particle trajectories downstream and has the potential to modify the signatures of the collisionless shock.
\end{abstract}

Collisionless shocks have been studied for many decades, mainly in the context of space and astrophysics [1-4]. Recently, shock acceleration raised significant interest in the quest for a laser-based ion acceleration scheme due to an experimentally demonstrated high beam quality [5-8]. Interpenetrating plasma slabs of hot electrons and cold ions are acting to set up the electrostatic fields via longitudinal plasma instabilities. The lighter electrons leaving the denser regions are held back by the electric fields, which pull the ions. Particles are trapped in the associated electrostatic potential, which steepens and eventually reaches a quasisteady-state collisionless electrostatic shock. Most of the theoretical work dates back to the 1970s [9-13] relying on the pseudo-Sagdeev potential [14] and progress has been mainly triggered by kinetic simulations [15-18].

The short formation time scales and the one dimensionality of the problem make it easily accessible with theory and computer simulations. However, long time shock evolution was often one dimensional or electrostatic codes were used, and the role of electromagnetic modes was mostly neglected. More advanced multidimensional simulations have shown the importance of electromagnetic modes also in this context, due to transverse modes which are excited on the ion time scale $[19,20]$. We show that in the case of very high electron temperatures associated with the formation of electrostatic shocks [21], electromagnetic modes become important on electron time scales, creating strong magnetic fields in the downstream of the shock.

With the increase in laser energy and intensity, the possibility to drive electrostatic shocks has become important for laboratory experiments of electrostatic shocks. Recent laser-driven shock experiments showed the appearance of an electromagnetic field structure [22-24], which was attributed to the ion-filamentation instability [25] that evolves on time scales of ten thousands of the inverse electron plasma frequency, $\omega_{p e}^{-1}$. As a main outcome of this Letter, we show that these structures can already be seeded and produced on tens of $\omega_{p e}^{-1}$ and remain in a quasisteady state over thousands of $\omega_{p e}^{-1}$. In fact, on electron time scales, the magnetic field is driven by the pure Weibel instability [26-28] due to a strong temperature anisotropy [29] which is caused by electron trapping in the downstream region of electrostatic shocks.

We will start by considering shock formation in a system of symmetric charge and current neutral counterstreaming beams, each consisting of a population of hot electrons and cold ions. The fluid velocities are chosen low enough and the electron temperature sufficiently high, so that the electron filamentation instability associated with the counterflows evolves only on time scales orders of magnitude larger than the shock formation time scale, according to the analysis in Ref. [21]. In this case, and for nonflow velocities, the shock formation process is dominated by electrostatic modes.

The initial stage of the shock formation process is studied in particle-in-cell simulations with the fully relativistic code OSIRIS [30,31]. We use a 3D simulation box with the length in each direction being $L_{x}=L_{y}=L_{z}=60 c / \omega_{p e}$, periodic boundaries in the transverse directions, and a spatial resolution $\Delta x=0.1 c / \omega_{p e}$ in all three directions. The temporal resolution is $\Delta t \omega_{p e}=0.057$, and a cubic interpolation scheme was used with four particles per cell and per species. Test runs with a higher number of particles per cell were also performed, yielding similar results. The full shock formation for a fluid of hot electrons and cold ions with proper velocities $u_{0}=\beta_{0} \gamma_{0}= \pm 0.015$, where $\beta_{0}=v_{0} / c$ and upstream Lorentz factor $\gamma_{0}=\left(1-v_{0} / c\right)^{-1 / 2}$ happens on time scales $t \gtrsim 10 \omega_{p i}^{-1}$, which we followed in $3 \mathrm{D}$ for a 
reduced mass ratio $m_{p} / m_{e}=100$. Long-term simulations with a realistic proton-to-electron mass ratio $m_{p} / m_{e}=1836$ were performed in two spatial dimensions up to $t \approx 10^{4} \omega_{p e}^{-1}$ with $L_{x}=10^{3} c / \omega_{p e}, L_{y}=450 c / \omega_{p e}, \Delta x=\Delta y=0.1 c / \omega_{p e}$, and $\Delta t \omega_{p e}=0.07$. In the 2D setup, the thermal parameter is $\Delta \gamma=k_{B} T_{e} / m_{e} c^{2}=20$, whereas it could be reduced to $\Delta \gamma=0.015$ in the 3D case due to the lower mass ratio, guaranteeing the electrostatic character of the shock [21].

In this configuration, similar to the configuration employed in recent experiments [6,7], two symmetric shocks moving in opposite directions (along $x$ ) are launched from the contact discontinuity at the center of the simulation box, where the two plasma shells initially come in contact. The region between the two shocks defines the downstream of the two nonlinear structures. Early in the shock formation process, we observe the generation of a magnetic field in the downstream region between the two shock fronts. This is illustrated in Fig. 1, where the field structure is presented after the electrostatic shocks have reached a quasisteady state. A strong longitudinal electric field has already formed at the shock front. At the same time, a strong perpendicular magnetic field has been generated, which is well confined to the downstream region of the shock. Unlike Weibel mediated shocks [4], the magnetic field in the shock front and in the upstream region is very small. The filamentary field structure in the downstream region indicates the Weibel instability as the driving mechanism, reinforced by the time scales of the process ( $\sim$ tens of $\omega_{p e}^{-1}$ ) and the transverse length scale of the filaments early in time $\left(\sim a\right.$ few $\left.c / \omega_{p e}\right)$.

Several 2D simulations with proper velocities $u_{0}=$ $0.005-0.1$ and $\Delta \gamma=0.01-20$, corresponding to electron thermal energies $k_{B} T_{e}$ in the range $\sim 5 \mathrm{keV}$ to $10 \mathrm{MeV}$
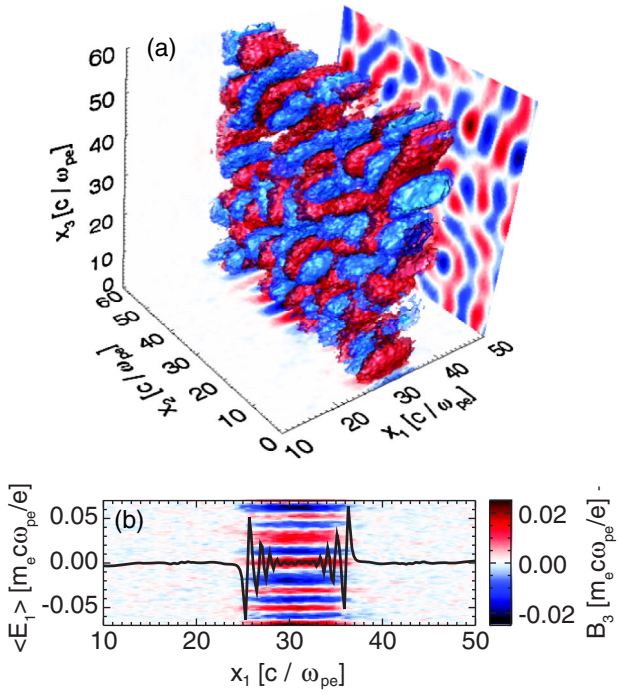

FIG. 1 (color online). Shock formation in the 3D simulation for mass ratio $m_{p} / m_{e}=100, u_{0}= \pm 0.015$, and $\Delta \gamma=0.015$ : (a) Perpendicular electromagnetic field, (b) box-averaged electrostatic field $\left\langle E_{1}\right\rangle$ (black), and 2D slice of magnetic field in (a) at $z=30 c / \omega_{p e}$ showing the extension of the filaments. Time is $t \omega_{p e}=460$. were performed in order to study the magnetic field formation process in electrostatic shocks in more detail. This parameter range covers astrophysical conditions, e.g., with estimated quasar temperatures of $\sim 10^{7} \mathrm{~K}$ [32], or laser-plasma interactions, where hot electrons can easily be generated with $T_{e \text {,hot }}$ up to several MeV.

The 2D simulations reproduce the same magnetic field generation mechanism with the field confined to the downstream region of the shock. The magnetic field energy averaged over $x_{2}$ in the center of the shock downstream region is represented in Fig. 2(a) for $u_{0}=0.1$ and $\Delta \gamma=10$. After a linear increase, at $t \approx 300 \omega_{p e}^{-1}$ the field growth saturates, and a quasisteady value $\varepsilon_{B} \approx 0.01 \varepsilon_{0} \approx 0.002\left(\varepsilon_{0}+\right.$ $\left.\varepsilon_{t h}\right)$ is reached, where $\varepsilon_{0}=n_{0} m_{p}\left(\gamma_{0}-1\right) c^{2}$ represents the kinetic energy density of the ions, $\varepsilon_{t h} \approx 3 \Delta \gamma / 2$ is the thermal energy density of the electrons, and $\varepsilon_{B}$ is the magnetic field energy density. This field structure can then seed the filamentation on the longer ion time scale and, thus, sustain a high level of $\epsilon_{B}$ covering the full downstream region for times at least as long as $t \approx 10^{4} \omega_{p e}^{-1}$.

We now analyze the different instabilities that can arise in initially unmagnetized counterstreaming electron-ion flows. For our range of parameters, the electron current filamentation instability is suppressed since the flows are hot $[33,34]$. Moreover, the cold ion-ion-filamentation instability, which has been considered in connection with recent experiments, has a maximum theoretical growth rate $\sigma_{i}=\sqrt{\left(2 / \gamma_{0}\right)} \beta_{0} \omega_{p i}=3.3 \times 10^{-3} \omega_{p e}$, and a saturation field $B \simeq m \gamma_{0}^{2} \sigma_{i}^{2} / q k_{i} u_{x 0}$ with $\gamma_{0}$ the Lorentz factor of the counterpropagating flows, proper velocity in the $x$ direction $u_{x 0}=\beta_{x 0} \gamma_{0}$, and wave number $k_{i}$ at the maximum growth rate $\sigma_{i}$ [34], yielding a saturated magnetic field of only $B=1.5 \times 10^{-3} m_{e} c \omega_{p e} / e$, clearly below the field values up to $B \approx 2 m_{e} c \omega_{p e} / e$ observed in the simulations. It is then clear that only an instability associated with the shock formation process can lead to magnetic field generation on the relevant time scales.
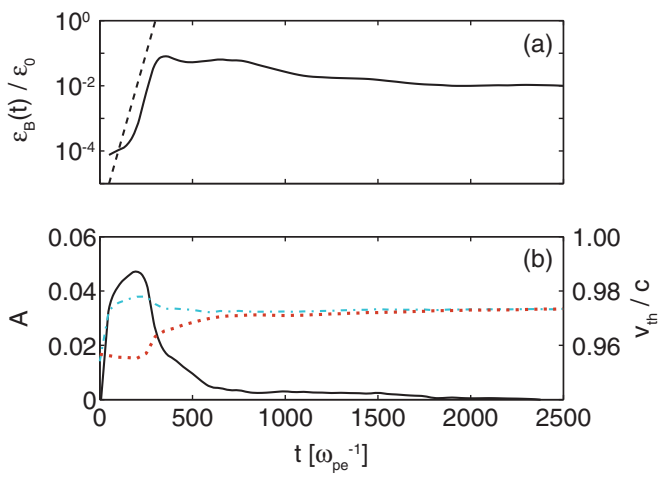

FIG. 2 (color online). Temporal evolution of (a) normalized magnetic energy density and (b) thermal velocities $v_{\text {th, }}$ (dash dotted), $v_{\text {th }, \perp}$ (dotted), and anisotropy $A$ (solid) in a 2D simulation with $m_{p} / m_{e}=1836, u_{0}= \pm 0.1$, and $\Delta \gamma=10$ measured over $\Delta x_{1}=0.7 c / \omega_{p e}$ at the center of the simulation box. The black dashed line in (a) is $\exp \left(2 \sigma_{m} t\right)$. 
We attribute the magnetic field growth and saturation level to the temperature anisotropy that is generated during the electrostatic shock formation process. This is illustrated by Fig. 2(b) showing the parallel and perpendicular thermal velocities of the downstream electron distribution, $v_{\text {th, } \|}$ and $v_{\text {th, } \perp}$, respectively, with $v_{\text {th }, \alpha}=\sqrt{k_{B} T_{\alpha} / m_{e}}$, together with the anisotropy parameter $A=\left(v_{\mathrm{th}, \|} / v_{\mathrm{th}, \perp}\right)^{2}-1$. In fact, the nonlinear evolution of the longitudinal modes associated with shock formation increase the electron temperature in the shock propagation direction due to wave breaking and electron trapping, while the transverse profile of the distribution function stays almost unchanged. At the time when the anisotropy reaches its maximum $A=0.048$, the magnetic field grows exponentially at its maximum growth rate, according to the theory for the Weibel instability [26]. Unlike previous works, which have addressed current filamentation scenarios (with free energy for the instability associated with nonzero fluid velocities of the flows), in this region the electron fluid velocity is zero, and the magnetic field originates only from the temperature anisotropy associated with preferential heating along the shock formation direction $x$ and the distortion of the distribution function due to electron trapping in the shock downstream.

We now quantify the main features of the instability driven by the electrons in the downstream of the electrostatic shock. In our model, we assume only the initial conditions of the flows and that an electrostatic shock is formed $[15,35]$. The growth rate can be calculated theoretically from first principles. Starting from the Sagdeev description for electrostatic shocks, the electron distribution function along the entire shock structure is calculated by considering a free, streaming population of electrons $f_{e \pm}=n_{0} \exp \left\{-\left[\gamma_{0}(\gamma-\varphi)-1 \pm u_{0} \sqrt{\left(\sqrt{1+u_{x}^{2}}-\varphi\right)^{2}-1}\right] /\right.$ $\Delta \gamma\}$, as well as a trapped population in the electrostatic potential $\varphi$ represented by a plateau in phase space for $\left.\left|u_{x}\right| \leq \sqrt{(1+\varphi)^{2}-1}, \quad f_{e, t}=n_{0} \exp \left\{-\left[\gamma_{0} \gamma_{\perp}-1\right]\right\} / \Delta \gamma\right\}$ with normalization factor $n_{0}$ [35]. This distribution function is then used to evaluate the dispersion relation for electromagnetic waves $k^{2} c^{2}-\omega^{2}-\omega_{p e}^{2}\left(U_{e}+V_{e}\right)=0$ with
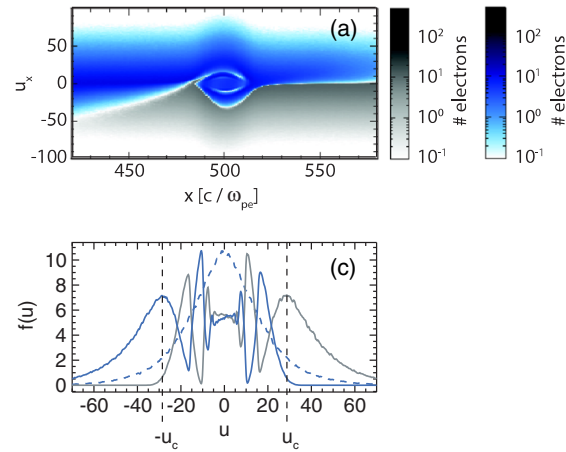

$$
\begin{aligned}
U_{e} & =\int_{-\infty}^{\infty} d^{3} u \frac{u_{x}}{\gamma} \frac{\partial f}{\partial u_{x}}, \\
V_{e} & =\int_{-\infty}^{\infty} d^{3} u \frac{u_{x}^{2}}{\gamma\left(\gamma \omega / k c-u_{x}\right)} \frac{\partial f}{\partial u_{z}} .
\end{aligned}
$$

In the nonrelativistic limit, we obtain [21]

$$
\begin{aligned}
& k^{2} c^{2}+\sigma^{2}+\omega_{p e}^{2}\left[1-V(\phi)\left[1+\frac{l \sigma}{\sqrt{2} v_{\mathrm{th}} k c} Z\left(\frac{l \sigma}{\sqrt{2} v_{\mathrm{th}} k c}\right)\right]\right] \\
& =0
\end{aligned}
$$

where $\sigma$ is the imaginary part of the wave frequency, $k$ is the wave number along the perpendicular direction, the plasma dispersion function is $Z$ [36], and $\phi=e \varphi / m_{e} c^{2}$ is the normalized electrostatic potential with $V(\phi)=n_{0}\left\{e \sqrt{\phi} / v_{\text {th }} \operatorname{erfc}\left[\sqrt{\phi} / v_{\text {th }}\right]+2 \sqrt{\phi / \pi v_{\text {th }}^{2}}+\right.$ $\left.\frac{4}{3} \sqrt{\phi^{3} / \pi} v_{\text {th }}^{-3} \exp \left[-v_{0}^{2} / 2 v_{\text {th }}^{2}\right]\right\}$ the Sagdeev potential and $n_{0}=\left[e^{\sqrt{\phi} / v_{\text {th }}} \operatorname{erfc}\left[\sqrt{\phi} / v_{\text {th }}\right]+2 \sqrt{\phi / \pi v_{\text {th }}^{2}} \exp \left[-v_{0}^{2} / 2 v_{\text {th }}^{2}\right]\right]^{-1}$ the electron density along the shock front. At the time when the magnetic field starts to grow, the electrostatic potential in the downstream region is approximately of the order of the initial ion kinetic energy [37], which leads to a maximum growth rate $\sigma_{m}=0.053 \omega_{p e}$ from Eq. (2) and matches well the simulation result in Fig. 2(a). The dominant wave number $k_{m}=0.14 \omega_{p e} / c$ corresponds to the wavelength $\lambda_{m}=2 \pi / k_{m}=45 c / \omega_{p e}$, which matches with the transverse spatial scale of the magnetic filaments at $t \omega_{p e} \leq 450$.

The analysis of the electron distribution function explains the origin of the anisotropy, which gives rise to the generation of the (electro)magnetic modes. The expansion of the hot electrons relative to the slower ions creates the strong space charge fields at the shock front. Particles are trapped in the field potential, leading to the formation of a vortex structure in phase space as seen in Fig. 3(a), which resembles the electron holes observed in previous simulations [38-41]. Along the parallel (三 shock propagation) direction, the two populations of initially thermal counterstreaming beams have broadened and start mixing, while
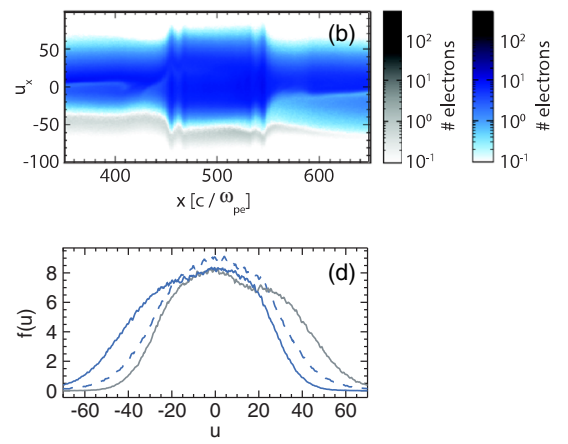

FIG. 3 (color online). Electron phase spaces $\left(u_{x}, x\right)$ of left beam with $u_{0}=0.1$ (blue) and right beam with $u_{0}=-0.1$ (gray) (a),(b) and electron distribution functions measured at $x=500 \mathrm{c} / \omega_{p e}$ for momentum $u_{x}$ (solid lines) for left and right beams in blue and gray (c), (d), respectively, for the 2D simulations shown in Fig. 2 and for $t \omega_{p e}=100$ (a),(c) and 1000 (b),(d). The dashed lines indicate the distribution of the transverse momentum $u_{y}$. 

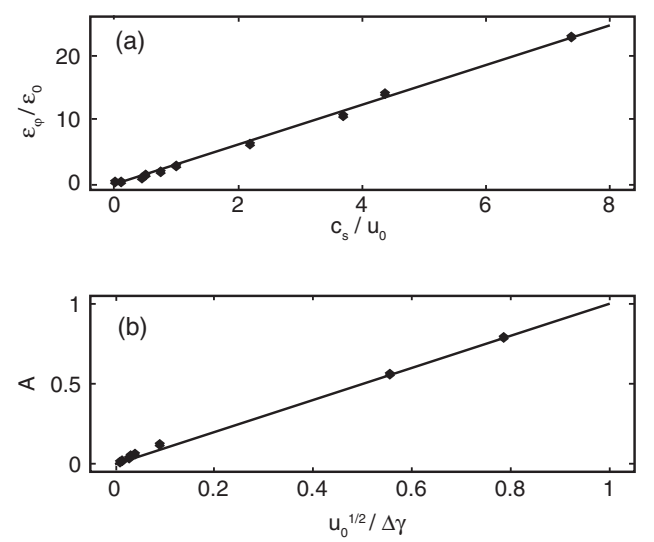

FIG. 4. Parameter scan of the (a) maximum energy of the electrostatic potential normalized to the upstream proton kinetic energy obtained from the simulations (the solid line represents a linear fit with $c_{s} / u_{0}$ ), and (b) associated maximum anisotropy $A$ (with linear fit $\sqrt{u_{0}} / \Delta \gamma$ ) for 2D simulations with $m_{p} / m_{e}=1836$.

the distribution in the perpendicular direction stays almost unchanged [Fig. 3(c)]. This structure with a flat distribution profile around $u_{1}=0$ and peaks at $\pm u_{c}$ remains unaltered over hundreds of $\omega_{p e}^{-1}$ [Figs. 3(b) and 3(d)]. At $t \approx 1000 \omega_{p e}^{-1}$, the electron distribution functions are close to thermalization [Figs. 2(b) and 3(d)], and the magnetic field energy has reached its quasisteady state [Fig. 2(a)].

To understand the dependence of the magnetic field generation on the properties of the flow, we have explored the dependence of the distribution function anisotropy $A$ after shock formation on the flow parameters $\Delta \gamma$ and $u_{0}$. Figure 4 shows the scaling of the electrostatic potential and the anisotropy with the upstream plasma parameters $\Delta \gamma$ (or $c_{s}=\sqrt{\Delta \gamma m_{e} / m_{p}}$ ) and $u_{0} \approx \sqrt{2\left(\gamma_{0}-1\right)}$ in the nonrelativistic limit. Here, the parameters have been extended beyond the electrostatic shock formation condition $v_{0} / c_{s} \lesssim 3$, for which the electrostatic potential is not strong enough to form a steady-state electrostatic shock.

It can be observed from Fig. 4(a) that the electrostatic potential increases with the electron temperature and with the bulk velocity of the upstream, $e \varphi \propto c_{s} / u_{0} \propto \sqrt{\Delta \gamma\left(\gamma_{0}-1\right)}$ [21]. Since the ion kinetic energy is a function of the initial Lorentz factor $\left[\propto\left(\gamma_{0}-1\right)\right]$, the normalization with the initial ion energy provides a scaling $e \varphi / \epsilon_{0} \propto \sqrt{\Delta \gamma /\left(\gamma_{0}-1\right)}$, meaning that the ability of ion reflection from the electrostatic potential decreases with the initial upstream velocity. The anisotropy in Fig. 4(b) shows an opposite trend; it increases with the fluid velocity and decreases with the electron temperature with a linear dependence on $\sqrt{u_{0}} / \Delta \gamma$. Although the electrostatic potential increases drastically with the electron temperature in the case of electrostatic shocks, particle trapping is apparently less efficient.

The above simulations were performed for the scenario when the two beams were initially in contact and penetrated as soon as the simulation started. We also considered the case where the flows were separated by a vacuum region of $200 c / \omega_{p e}$ to model the collision of two initially separated flows, as occurring in experimental setups, taking into account the plasma expansion into vacuum. In this case, a shock is also formed due to an electrostatic field between the hot electrons and cold ions, and the generation of a magnetic field in the downstream is observed with the same driving mechanism. In the case of beams in contact the anisotropy is higher; the amplification level after saturation is the same, but the separation leads to a decrease of the magnetic field growth rate by a factor 15 .

We note that the generation mechanism for magnetic fields in the case of electrostatic shocks is fundamentally different from the current-filamentation-driven amplification in the case of electromagnetic shocks. In the first case, it is actually the original Weibel instability, which is powered by a temperature anisotropy closely tied to the shock formation process and the steady-state shock structure. Therefore, the magnetic field appears in the hotter downstream region of the electrostatic shock (see Fig. 5). While on the other hand, in the electromagnetic case, it is the fresh, cold upstream plasma that drives the instability, and the magnetic field appears close to the shock front, as illustrated in Fig. 5, which shows a Weibelmediated electromagnetic shock for $\gamma_{0}=20$ and $\Delta \gamma=$ $10^{-3}$ (parameters for both cases determined from Ref. [21]).

In conclusion, we showed that strong magnetic fields are generated in the downstream region of electrostatic shocks. Because of strong particle trapping in the downstream region, a temperature anisotropy is generated in the electron
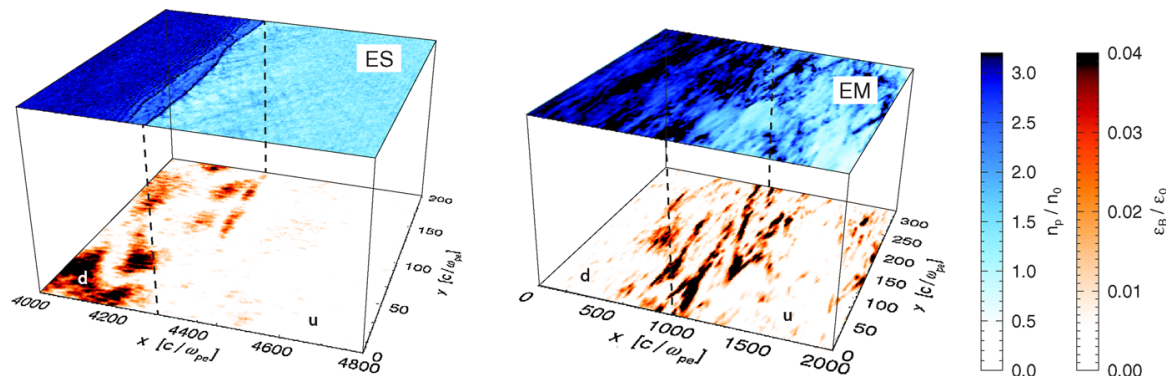

FIG. 5 (color online). Proton density (blue) and normalized magnetic field energy density (orange) obtained from 2D simulations for an electrostatic shock (ES) and a Weibel-mediated shock (EM). The dashed lines indicate the shock front with the transition from the upstream (u) to the downstream region $(\mathrm{d})$. 
distribution function, which gives rise to electromagnetic Weibel modes. The field clearly forms on electron time scales, and the growth rates captured in the simulations match the theoretical predictions and with the generated field amplitude orders of magnitude higher than would be expected from the ion-ion-filamentation instability. This field can then seed other electromagnetic instabilities occurring on longer time scales. We have followed the field evolution in 2D and 3D simulations and showed that a quasi-steady-state value is reached, with the magnetic field being generated only in the downstream region, in contrary to electromagnetic shocks where the filamentation instability creates a magnetic field across the shock front. We have observed that since the field is generated in the downstream region, the effect of the selfgenerated magnetic field on the formation process is negligible, and the properties of the electrostatic shock, e.g., in terms of ion reflection, are preserved $[7,8,42]$. On the other hand, the strong field in the downstream region influences the dynamics of the particles in this region, and it can lead to distinct signatures of the shock. On the quest for the generation of collisionless shocks in the laboratory mediated by magnetic fields, we conjecture that the identification of the structures cannot rely only on the measurement of the self-generated magnetic fields. Experiments will have to take into account also the dynamics of the magnetic field generated by this mechanism in electrostatic shocks. Thus, identification of electromagnetic shocks, in opposition to electrostatic shocks, should rely instead on a different approach based on the direct measurement of the relative importance of the longitudinal electric field in comparison with the the magnetic fields along the shock front.

This work was partially supported by the European Research Council (ERC-2010-AdG Grant No. 267841) and FCT (Portugal) Grants No. SFRH/BPD/65008/2009, No. SFRH/BD/38952/2007, and No. PTDC/FIS/111720/ 2009. The authors gratefully acknowledge PRACE for providing access to SuperMUC based in Germany at the Leibniz research center. We also acknowledge the Gauss Centre for Supercomputing (GCS) for providing computing time through the John von Neumann Institute for Computing (NIC) on the GCS share of the supercomputer JUQUEEN at Jülich Supercomputing Centre (JSC).

*anne@tp4.rub.de

[1] R. Z. Sagdeev and C. F. Kennel, Sci. Am. 264, No. 4, 106 (1991).

[2] A. Spitkovsky, Astrophys. J. 682, L5 (2008).

[3] S. F. Martins, R. A. Fonseca, L. O. Silva, and W. B. Mori, Astrophys. J. 695, L189 (2009).

[4] F. Fiuza, R. A. Fonseca, J. Tonge, W. B. Mori, and L. O. Silva, Phys. Rev. Lett. 108, 235004 (2012).

[5] S. S. Bulanov et al., Med. Phys. 35, 1770 (2008).

[6] L. Romagnani et al., Phys. Rev. Lett. 101, 025004 (2008).

[7] D. Haberberger, S. Tochitsky, F. Fiuza, C. Gong, R. A. Fonseca, L. O. Silva, W. B. Mori, and C. Joshi, Nat. Phys. 8, 95 (2012).
[8] F. Fiuza, A. Stockem, E. Boella, R. A. Fonseca, L. O. Silva, D. Haberberger, S. Tochitsky, C. Gong, W. B. Mori, and C. Joshi, Phys. Rev. Lett. 109, 215001 (2012).

[9] D. Montgomery and G. Joyce, J. Plasma Phys. 3, 1 (1969).

[10] D. Biskamp, J. Plasma Phys. 3, 411 (1969).

[11] D. W. Forslund and C. R. Shonk, Phys. Rev. Lett. 25, 1699 (1970).

[12] D. A. Tidman and N. A. Krall, Shock Waves in Collisionless Plasmas (Wiley-Interscience, New York, 1971).

[13] H. Schamel, Plasma Phys. 14, 905 (1972).

[14] R. Z. Sagdeev, Rev. Plasma Phys. 423 (1966).

[15] G. Sorasio, M. Marti, R. Fonseca, and L. O. Silva, Phys. Rev. Lett. 96, 045005 (2006).

[16] J. Denavit, Phys. Rev. Lett. 69, 3052 (1992).

[17] L. O. Silva, M. Marti, J. R. Davies, and R. A. Fonseca, Phys. Rev. Lett. 92, 015002 (2004).

[18] M. E. Dieckmann, G. Sarri, L. Romagnani, I. Kourakis, and M. Borghesi, Plasma Phys. Controlled Fusion 52, 025001 (2010).

[19] G. Sarri, M. E. Dieckmann, I. Kourakis, and M. Borghesi, Phys. Rev. Lett. 107, 025003 (2011).

[20] T. N. Kato and H. Takabe, Phys. Plasmas 17, 032114 (2010).

[21] A. Stockem, F. Fiuza, A. Bret, R. A. Fonseca, and L. O. Silva, Sci. Rep. 4, 3934 (2014).

[22] N. L. Kugland et al., Nat. Phys. 8, 809 (2012).

[23] W. Fox, G. Fiksel, A. Bhattacharjee, P.-Y. Chang, K. Germaschewski, S. X. Hu, and P. M. Nilson, Phys. Rev. Lett. 111, 225002 (2013).

[24] C. M. Huntington et al., arXiv:1310.3337.

[25] H.-S. Park et al., High Energy Density Phys. 8, 38 (2012).

[26] E. S. Weibel, Phys. Rev. Lett. 2, 83 (1959).

[27] B. D. Fried, Phys. Fluids 2, 337 (1959).

[28] A. Bret, Astrophys. J. 699, 990 (2009).

[29] C. Thaury, P. Mora, A. Heron, J. C. Adam, and T. M. Antonsen, Phys. Rev. E 82, 026408 (2010).

[30] R. A. Fonseca, L. O. Silva, F. S. Tsung, V. K. Decyk, W. Lu, C. Ren, W. B. Mori, S. Deng, S. Lee, T. Katsouleas, and J. C. Adam, Lect. Notes Comput. Sci. 2331, 342 (2002).

[31] R. A. Fonseca, S. F. Martins, L. O. Silva, J. W. Tonge, F. S. Tsung, and W. B. Mori, Plasma Phys. Controlled Fusion 50, 124034 (2008).

[32] P. B. Hall et al., arXiv:1308.6010.

[33] J. J. Su, T. Katsouleas, J. M. Dawson, P. Chen, M. Jones, and R. Keinigs, IEEE Trans. Plasma Sci. 15, 192 (1987).

[34] L. O. Silva, R. A. Fonseca, J. W. Tonge, W. B. Mori, and J. M. Dawson, Phys. Plasmas 9, 2458 (2002).

[35] A. Stockem, E. Boella, F. Fiuza, and L. O. Silva, Phys. Rev. E 87, 043116 (2013).

[36] B. D. Fried and S. D. Conte, The Plasma Dispersion Function (Academic, New York, 1961).

[37] When the shock is formed, the upstream ions are reflected by the electric field in the shock front.

[38] M. E. Dieckmann, P. K. Shukla, and L. O. C. Drury, Mon. Not. R. Astron. Soc. 367, 1072 (2006).

[39] M. E. Dieckmann, Nonlinear Processes Geophys. 15, 831 (2008).

[40] M. V. Goldman, D. L. Newman, and P. Pritchett, Geophys. Res. Lett. 35, L22109 (2008).

[41] C.-R. Choi, K.-C. Rha, C.-M. Ryu, E.-J. Choi, K.-W. Min, E. Lee, and G. K. Parks, Phys. Plasmas 19, 102903 (2012).

[42] F. Fiuza, A. Stockem, E. Boella, R. A. Fonseca, L. O. Silva, D. Haberberger, S. Tochitsky, W. B. Mori, and C. Joshi, Phys. Plasmas 20, 056304 (2013). 